\title{
Prospective Assessment of Elevated Serum Bilirubin Level in Diagnosis of Acute Appendicitis
}

\author{
Ahmed Abdel-Fattah Elshoura, Mohamed Hamdy Abo-Ryia, Ayman Elsadany, \\ Sherif Abd-Al Fattah Saber, Gamal Ibrahim Mousa
}

General Surgery Department, Faculty of Medicine, Tanta University, Tanta, Egypt

Email address:

ahmed.elashora28@hotmail.com (A. Abdel-Fattah E.), mhaboryiah@gmail.com (M. H. Abo-Ryia),

sherifsaber1980@gmail.com (S. Abd-Al F. S.)

\section{To cite this article:}

Ahmed Abdel-Fattah Elshoura, Mohamed Hamdy Abo-Ryia, Ayman Elsadany, Sherif Abd-Al Fattah Saber, Gamal Ibrahim Mousa. Prospective Assessment of Elevated Serum Bilirubin Level in Diagnosis of Acute Appendicitis. Advances in Surgical Sciences.

Vol. 6, No. 1, 2018, pp. 27-30. doi: 10.11648/j.ass.20180601.15

Received: April 28, 2018; Accepted: May 22, 2018; Published: June 12, 2018

\begin{abstract}
Background: The rate of misdiagnosis of appendicitis has remained constant, despite the advance in diagnostic modalities. So, the search for a reliable marker is necessary. The aim of this study was to determine the value of hyperbilirubinemia as a marker for acute appendicitis. Patients and Methods: This prospective study was carried out during the period from April 2014 till April 2017 and included 369 patients presented with right iliac fossa pain diagnosed clinically as acute appendicitis and submitted to appendectomy (open or laparoscopic). Demographic data, data of clinical examination, radiological and laboratory investigations (specifically, complete blood count (CBC), C- reactive protein (CRP) and total serum bilirubin) were collected. Then appendectomy was done, the removed appendices were sent for histopathological examination. Patients were divided into three groups: group (I) histopathologically normal appendix, group (II) simple appendicitis and group (III) Complicated appendicitis. The data of the three groups were analyzed and compared. Results: This study included 369 patiens, $195(52.8 \%)$ are males. Mean age was $22.6 \pm 7.22$ years. Open appendectomy was performed in $306(91.2 \%)$ patients and laparoscopic in 63 (8.8\%). Histopathological examination revealed normal appendix in 69 (18.6\%) patients, simple appendicitis in $240(65.0 \%)$ and complicated appendicitis in $60(16.2 \%)$. The serum total bilirubin had a higher specificity (87\%) compared with white blood cells (WBC) $(70 \%)$ and CRP $(65 \%)$, but a lower sensitivity $(28 \%$ vs. $66 \%$ and $58 \%$, respectively) for acute appendicitis versus normal appendix group and a higher specificity $(80 \%)$ than both WBC (35\%) and CRP (45\%), but a lower sensitivity than WBC and CRP (60\% vs. $70 \%$ and $70 \%$ respectively) for complicated appendicitis versus simple appendicitis. Conclusion: Hyperbilirubinemia is a significant predictor in diagnosis of acute appendicitis in cases of right iliac fossa pain. It is also a significant predictor of complication (perforation and gangrene) in cases of acute appendicitis.
\end{abstract}

Keywords: Appendicitis, Hyperbilirubinemia, Sensitivity, Specificity

\section{Introduction}

Acute appendicitis is one of the most common surgical conditions with the life time risk for appendectomy is estimated to be $12 \%$ for males and $23 \%$ for females. appendectomy is a minor procedure without significant consequences, when diagnosed early. However, delayed diagnosis with subsequent appendicular perforation leads to an increased rate of postoperative morbidity and mortality [1].
Despite the remarkable advancement in diagnostic modalities like high resolution computed tomography (CT), still the rate of misdiagnosis is remaining about $15 \%$ [2].

This urges the search for more consistent markers that can differentiate between complicated \& uncomplicated acute appendicitis. Hyperbilirubinemia is frequently observed in patients with septic foci. In the meanwhile, acute appendicitis is the most common intra-abdominal septic focus in surgical 
patients with E. coli and Bacteroides fragilis are the most frequent causative organisms. Multiple retrospective studies have shown a high specificity of hyperbilirubinemia in perforated appendicitis. The underlying mechanism might be related to impaired hepatic conjugation \& biliary excretion of bilirubin by bacterial endotoxins [3].

It was reported that, in presence of septic foci, systemic \& intrahepatic pro-inflammatory cytokines increase that lead to impairment of bile production and flow. Also the increase in nitric oxide and decrease in aquaporins in presence of sepsis impair bile flow [4].

The aim of this study was to prospectively assess the role of elevated serum bilirubin level in diagnosis of acute appendicitis.

\section{Patients and Methods}

This study was carried out at the general surgery department, Tanta university hospital, Tanta, Egypt, during the period from April 2014 till April 2017 and included 369 patients presented with right iliac fossa pain diagnosed clinically as acute appendicitis and submitted to appendectomy (open or laparoscopic). Patient with history of liver disease that could lead to deranged liver function tests (LFTs), patients with hemolytic diseases and patients who were managed conservatively were excluded. Demographic data, data of clinical examination, radiological and laboratory investigations (specifically, CBC, CRP and total serum bilirubin) were collected. Then appendectomy either by open or laparoscopic surgery was done and the removed appendices were sent for histopathological examination. According to the operative findings and the results of histopathological examination of the appendices, patients were divided into the following three groups: group (I) histopathologically normal appendix, group (II) Inflamed non-complicated appendix (simple appendicitis) and group (III) Complicated appendicitis (perforated or gangrenous). The data of the three groups were analyzed and compared.

The collected data were tabulated and analyzed statistically using SPSS software, version 20 (SPSS Inc., Chicago, USA). One way analysis of variance ANOVA was used to compare means of quantitative date between the three groups. While Chi square test was performed for nonparametric data as a test of significance. Tests of Validity of test parameter were calculated as following:

Sensitivity $=$ True Positives $\div$ (True Positives + False Negatives).

Specificity $=$ True Negatives $\div$ (True Negatives + False Positives).

Positive Predictive Value $=$ True Positives $\div$ (True Positives +False Positives).

Negative Predictive Value $=$ True Negatives $\div$ (True Negatives +False Negatives).

\section{Results}

This study included 369 patients, $195(52.8 \%)$ males and $174(47.2 \%)$ females with a mean age of $22.6 \pm 7.22$ years. Most patients $(60.2 \%)$ were under the age of 20 year. The body mass index ranged from $23.5-35 \mathrm{~kg} / \mathrm{m}^{2}$ with a mean of $27.2 \pm 1.65 \mathrm{~kg} / \mathrm{m}^{2}$. Open appendectomy was performed in 306 $(91.2 \%)$ patients and laparoscopic appendectomy in 63 $(8.8 \%)$ patients. Histopathological examination revealed normal appendix in $69(18.6 \%)$ patients, simple appendicitis in $240(65.0 \%)$ patients and complicated appendicitis in 60 $(16.2 \%)$ patients.

There was no significant difference between the three groups as regard; age and gender ( $p=0.125,0.342$ respectively). The mean duration of pain was significantly longer in the group of complicated appendicitis than the other two groups (simple appendicitis and normal appendix). It was $74.40 \pm 28.08,45.36 \pm 19.92,46.08 \pm 15.72$ hours respectively, $\mathrm{p}$ value $=0.005$. The mean value of WBCs count was significantly higher in the group of complicated appendicitis than the group of simple appendicitis and the group of normal appendices $(14.01 \pm 4.15,12.02 \pm 3.95,11.21 \pm 3.52$ respectively), $\mathrm{P}$ value $=0.025$.

The mean value of total serum bilirubin was significantly higher in the group of complicated appendicitis than the group of simple appendicitis and the group of normal appendices $(1.23 \pm 0.42,0.84 \pm 0.27,0.77 \pm 0.22)$ respectively, $p$ value of 0.002 . CRP was positive in $70 \%$ of cases in the group of complicated appendicitis versus $55 \%$ in the group of simple appendicitis and $34.87 \%$ in the group of normal appendices, $\mathrm{P}$ value $=0.002$.

Table 1. Sensitivity, specificity, positive /negative predictive values and diagnostic accuracy of serum bilirubin, WBC and CRP in differentiation between normal appendix and acute appendicitis, using pathological findings as the gold standard.

\begin{tabular}{llllll}
\hline Parameter & Sensitivity & Specificity & Positive predictive value & Negative predictive value & Accuracy \\
\hline Total Bilirubin & $28 \%$ & $87 \%$ & $90 \%$ & $30 \%$ & $39 \%$ \\
WBCs & $66 \%$ & $70 \%$ & $90 \%$ & $32 \%$ & $67 \%$ \\
CRP & $58 \%$ & $65 \%$ & $88 \%$ & $26 \%$ & $59 \%$ \\
\hline
\end{tabular}

Table 2. Sensitivity, specificity, positive /negative predictive values and diagnostic accuracy of serum bilirubin, WBC and CRP in differentiation between simple and complicated appendicitis, using pathological findings as the gold standard.

\begin{tabular}{|c|c|c|c|c|c|}
\hline Parameter & Sensitivity & Specificity & Positive predictive value & Negative predictive value & Accuracy \\
\hline Total Bilirubin & $60 \%$ & $80 \%$ & $43 \%$ & $69 \%$ & $76 \%$ \\
\hline WBCs & $70 \%$ & $35 \%$ & $21 \%$ & $82 \%$ & $42 \%$ \\
\hline CRP & $70 \%$ & $45 \%$ & $24 \%$ & $82 \%$ & $50 \%$ \\
\hline
\end{tabular}




\section{Discussion}

Acute appendicitis (AA) is the most frequent surgical emergency, associated with high incidence of morbidity and mortality if not managed early. Early diagnosis and appropriate early operative intervention brings favorable outcomes [5].

The diagnosis in the majority of cases is mainly by clinical examination and confirmed by laboratory and radiological investigations. However, diagnosis will be difficult sometimes even after performing all these tests [6]. And this may lead to either the removal of the patient's normal appendix or delay in diagnosis with its serious sequences like rupture, abscess and peritonitis that may cause death [5]. Several retrospective and some prospective studies have analyzed hyperbilirubinemia as a marker of acute appendicitis and perforation, but none of these studies has been sufficiently powered to analyze the precise sensitivity and specificity in diagnosis [7].

In this study, hyperbilirubinemia was found to be significantly associated with acute appendicitis groups versus normal appendix group and also, was statistically significant with complicated appendicitis versus simple appendicitis. The serum total bilirubin had a higher specificity $(87 \%)$ compared with WBCs count $(70 \%)$ and CRP $(65 \%)$, but a lower sensitivity ( $28 \%$ vs. $66 \%$ and $58 \%$, respectively) for acute appendicitis versus normal appendix group and a higher specificity $(80 \%)$ than both WBCs count $(35 \%)$ and CRP (45\%), but a lower sensitivity than WBCs count and CRP ( $60 \%$ vs. $70 \%$ and $70 \%$ respectively) for complicated appendicitis versus simple appendicitis.

This agrees with the findings of D'Souza et al who reported that hyperbilirubinemia was significantly associated with acute appendicitis versus normal appendix group and with complicated appendicitis versus simple appendicitis. Also, total bilirubin had a higher specificity $(96 \%)$ compared with WBCs count $(71 \%)$ and CRP (62\%), but a lower sensitivity ( $27 \%$ vs. $68 \%$ and $82 \%$ respectively) for acute appendicitis and a higher specificity $(82 \%)$ than both WBCs count (34\%) and CRP (21\%), but a lower sensitivity, for complicated appendicitis versus simple appendicitis [8].

Emmanuel et al reported that the mean total bilirubin levels were higher in patients with simple appendicitis compared to those with a non-inflamed appendix. That study reported a high number of patients with simple appendicitis having hyperbilirubinemia on admission $(30 \%$ vs. $12 \%$ in current study). In Emmanuel's study, hyperbilirubinemia showed specificity of $88 \%$, positive predictive value of $91 \%$ for acute appendicitis and Specificity of $70 \%$ for perforation or gangrene [2].

Sand et al found the specificity of hyperbilirubinemia for appendicular perforation to be $86 \%$ versus $55 \%$ and $35 \%$ for WBCs count and CRP, respectively. Whereas, the sensitivity was $70 \%$ for hyperbilirubinemia compared with $81 \%$ for WBCs count and $96 \%$ for CRP. That study stated that hyperbilirubinemia and clinical symptoms of appendicitis indicate a higher probability of appendiceal perforation in comparison to clinical symptoms only with normal bilirubin levels [9].

Giordano et al documented that hyperbilirubinemia had a sensitivity of $49 \%$, specificity of $82 \%$, positive and negative likelihood ratios of 2.51 and $58 \%$. Besides, receiving operating characteristic (ROC) curve and analysis for area under curve (AUC) of $73 \%$ as a predictor of perforation in acute appendicitis and concluded that hyperbilirubinemia with clinical features of appendicitis indicate severe acute appendicitis which should be managed by early appendectomy [3].

Burcharth et al reported that elevated serum total bilirubin was significantly higher in patients with appendiceal perforation compared to patients with simple appendicitis (sensitivity $38 \%-77 \%$ and specificity $70 \%-87 \%$ ). WBCs count and CRP showed a higher sensitivity $(88.7 \%$ and $83.6 \%$, respectively) \& a lower specificity $(71.9 \%$ and $57.8 \%$ respectively) for detection of acute appendicitis. In discrimination between simple and complicated appendicitis, elevated serum CRP showed higher specificity compared with elevated WBCs count $(70.3 \%$ vs. $65.8 \%)$ and lower sensitivity (80.6\%vs.91.8\% respectively) [10].

\section{Conclusion}

Hyperbilirubinemia is a significant predictor in diagnosis of acute appendicitis in cases of right iliac fossa pain. It is also a significant predictor of complication (perforation and gangrene) in cases of acute appendicitis. The combined estimation of $\mathrm{WBC}$, serum CRP and total bilirubin improves the diagnostic yield by combining the high sensitivity of WBC and CRP with the high specificity of total bilirubin, allowing early detection of cases who might develop complications and enabling better decision making.

\section{References}

[1] Ditillo MF, James DO, Dziura, et al. (2006) Is it Safe to Delay Appendectomy in Adults with Acute Appendicitia? Ann Surg, 244 (5): 656-660.

[2] Emmanuel A, Murchan P, Wilson I, et al. (2011) The value of hyperbilirubinaemia in the diagnosis of acute appendicitis. Ann R CollSurg Engl, 93 (3): 213-217.

[3] Giordano S, Paakkonen M, Salminen P, et al. (2013). Elevated serum bilirubin in assessing the likelihood of perforation in acute appendicitis: A diagnostic meta-analysis. International Journal of Surgery, 11: 795-800.

[4] Bohgal HK \& Sanyal AJ. (2013) The molecular pathogenesis of cholestasis in sepsis. Frontiers in Bioscience, 5 (1): 87-96.

[5] Subramanyam VV, Bangla G and Jyothi C. (2016) Serum bilirubin reliable diagnostic marker in detecting complicated appendicitis. J Evid Based Med Healthc, 3 (2): 66-71. 
[6] Kanumba ES, Mabula JB, Rambau P, et al. (2011) Modified Alvarado scoring system as a diagnostic tool for acute appendicitis at Bugando Medical Centre, Mwanza, Tanzania. BMC Surg, 11 (4): 1-5.

[7] McGowan DR, Sims HM, Zia K, et al. (2013) The value of biochemical markers in predicting a perforation in acute appendicitis. ANZ J Surg, 83: 79-83.

[8] D'Souza N, Karim D, Sunthareswaran R, et al. (2013) Bilirubin: a diagnostic marker for appendicitis. International Journal of Surgery, 11: 1114-1117.
[9] Sand M, Bechara FG, Holland-Letz T, et al. (2009) Diagnostic value of hyperbilirubinemia as a predictive factor for appendiceal perforation in acute appendicitis. Am. J. Surg, 198: 193-198.

[10] Burcharth J, Pommergaard HC, Rosenberg J, et al. (2013) Hyperbilirubinemia as a predictor for appendiceal perforation: a systematic review. Scand. J. Surg, 102: 55-60. 